\title{
A wide role for NOTCH1 signaling in acute leukemia
}

\author{
Raffaella Chiaramonte ${ }^{\mathrm{a}, *}$, Andrea Basile $^{\mathrm{a}}$, Elena Tassi ${ }^{\mathrm{a}}$, Elisabetta Calzavara $^{\mathrm{a}}$, \\ Valentina Cecchinato $^{\mathrm{a}}$, Vincenzo Rossi ${ }^{\mathrm{b}}$, Andrea Biondi ${ }^{\mathrm{b}}$, Paola Comi ${ }^{\mathrm{a}}$ \\ ${ }^{\mathrm{a}}$ Department of Biomedical Science and Technology, University of Milano, LITA via Fratelli Cervi 93, 20090 Segrate, Milano, Italy \\ ${ }^{\mathrm{b}}$ Centro M. Tettamanti, Clinica Pediatrica, University of Milano-Bicocca, S.Gerardo Hospital, Monza, Italy
}

Received 1 June 2004; received in revised form 18 July 2004; accepted 20 July 2004

\begin{abstract}
NOTCH1 is involved in the pathogenesis of T-acute lymphoblastic leukemia (T-ALL) carrying the very rare translocation $\mathrm{t}(7 ; 9)(\mathrm{q} 34 ; \mathrm{q} 34.3)$.

We analyzed the expression of genes belonging to NOTCH pathway, in acute leukemia primary samples and lymphoblastoid cell lines. NOTCH1 pathway activation represents a common feature of T-ALL when compared to acute myelogenous leukemia (AML) and B-cell precursor acute lymphoblastic leukemia. The contemporary expression of NOTCHI and its ligands on cell surface contributes to high levels of pathway activity. AML primary samples show high levels of JAGGED1 expression despite the low NOTCH1 pathway activation, consistent with an autonomous JAGGED1 signaling in myeloid leukemogenesis. (C) 2004 Elsevier Ireland Ltd. All rights reserved.
\end{abstract}

Keywords: NOTCH1; JAGGED1; T-ALL; BCP-ALL; AML

\section{Introduction}

NOTCH gene family encodes single pass transmembrane receptors able to transduce intercellular signals involved in cell-fate determination [1]. NOTCH1 signal is triggered by the Delta/Serrate/ Lag2 (DSL) ligands [1]. The DSL family of genes include the vertebrate homologues JAGGED1, JAGGED2, DLL-1 [1], DLL-3 and DLL-4 [2].

\footnotetext{
* Corresponding author. Tel.: + 39-025 033 0404/7/9; fax: + 390250330411.

E-mail address: raffaella.chiaramonte@unimi.it (R. Chiaramonte).
}

NOTCH pathway plays a role in haematopoiesis affecting both stem cells and committed progenitors [3]. The expression of NOTCH1 and its ligands in thymic stromal cells and in developing thymocytes is consistent with NOTCH function. NOTCH1 is turn on before T-cell commitment along the lymphoid development; a variety of model systems indicates that NOTCH1 signaling is required for the earliest stages of T-cell commitment in the thymus and that, in its absence, cells develop toward the B-lineage [4]. NOTCH1 regulates myeloid cells differentiation and safeguards them from apoptosis [5]. In myeloid environment, JAGGED1 appears to play a critical role as its enforced expression causes the loss of cell 
ability to differentiate following the stimulation with GM-GSF [6].

The fundamental role played by the NOTCH pathway in T-lineage proliferation and differentiation has a counterpart in the involvement of $\mathrm{NOTCH}$ signaling alterations in the pathogenesis of T-cell acute lymphoblastic leukemia (T-ALL). The $t(7 ; 9)$ (q34;q34.3) translocation, which occurs specifically in T-ALL, places the $3^{\prime}$ half of $N O T C H 1$ gene sequence downstream TCR- $\beta$ regulative sequence [7] and leads to the overexpression of a constitutively active intracellular portion of NOTCH1 (NICD) [8]. This form has been proved to be neoplastic in vivo and, if over expressed in mouse bone marrow, to induce selectively T-ALL [8] suggesting that NOTCHI behaves as a gate keeping gene in the T-lineage. Yan et al. [9] demonstrated that the induction of the $D L L-4$ gene expression in bone marrow cells gives rise to T-cell leukemia. Moreover, it has been proposed that the ligand JAGGED1 may be responsible of NOTCH activation found in T-cell-derived anaplastic large cell lymphoma by inducing proliferation and inhibiting apoptosis [10]. Regarding NOTCH signaling in myeloid neoplastic counterpart, acute myelogenous leukemia is characterized by abnormalities in the NOTCH1-JAGGED1 system which would give rise to excessive self-renewal and block of differentiation [11].

We have previously demonstrated that, independently from the translocation $\mathrm{t}(7 ; 9)(\mathrm{q} 34 ; \mathrm{q} 34.3)$, the NOTCH pathway is activated in T-ALL cell lines when compared to B-cell lymphomas (EBV negative) or to normal $\mathrm{B}$ and $\mathrm{T}$ lymphocytes from healthy donors [12].

In the present study we further extend these observations by analysing the expression of a large panel of genes involved in NOTCH pathway activity, in a series of primary leukemia cells isolated from bone marrow of pediatric patients with T-ALL, B cell precursor ALL (BCP-ALL) and AML. Our findings reinforce the notion that NOTCH pathway oversignaling represents a hallmark of the T-ALL since AML and BCP-ALL showed significantly lower levels of expression. Of note T ALL cell lines and primary samples are both characterized by the contemporary expression of NOTCH receptor and its ligands supporting the hypothesis that $\mathrm{NOTCH}$ pathway activation in T-ALL can be due, at least in part, to an auto-activation of this pathway. By contrast in AML we found high levels of JAGGED1 expression despite of the low expression of NOTCH1 pathway genes, consistent with a potential role of autonomous JAGGED1 signaling in myeloid leukemogenesis.

\section{Materials and methods}

\subsection{Cell cultures and blood samples}

Under informed consent by the parents or the guardians, 32 children with T-ALL, 25 with B-cell precursor ALL and 20 with AML were included in the study. All patients have been referred to a single Institution (Clinica Pediatrica of Monza, Italy) and treated according to the protocols of the 'Associazione Italiana Ematologia ed Oncologia Pediatrica' (AIEOP). Diagnoses have been made according to standard morphological, immunological and molecular genetics criteria. Peripheral blood mononuclear cells (PBMC) have been separated on Ficoll-Hypaque gradient, frozen as viable cells and kept at $-80^{\circ} \mathrm{C}$. Mononuclear cells isolated from peripheral blood of healthy volunteers were used as controls. The following T-ALL cell lines, Jurkat, SupT1, MOLT4, CEM, HuT78, RPMI-8402, FRO and KE37, and B-cell derived leukemia/lymphoma cell lines, BC2, Raji, AS283, VAL, Daudy, BL135, BL41, EW36, Ly8, were used. Cell lines were cultured as previously reported [12] in 5\% $\mathrm{CO}_{2}$ atmosphere in complete RPMI-1640 medium supplemented with $10 \%$ foetal calf serum.

\subsection{Retrotranscription and amplification}

Total RNA was extracted according to Chomczynski and Sacchi [13]. RNA samples were retrotranscripted by M-MLV Reverse Transcriptase (Gibco BRL-Life Technologies) in the conditions suggested. PCR was performed in a $20 \mu \mathrm{l}$ reaction mixture containing $2 \mu \mathrm{l}$ of cDNA, $1 \times$ PCR Buffer II, $1.5 \mathrm{mM} \mathrm{MgCl}_{2}, 200 \mu \mathrm{M}$ dNTPs, 0.25 units of AmpliTaq DNA Polymerase (Perkin-Elmer Corporation). Amplifications were performed for the number of cycles specified in Table 1 according to the following regime: denaturation at $94{ }^{\circ} \mathrm{C}$ for $30 \mathrm{~s}$, annealing at $57^{\circ} \mathrm{C}$ for $30 \mathrm{~s}$ and extension at $72{ }^{\circ} \mathrm{C}$ for $30 \mathrm{~s}$. 
Table 1

PCR primers and number of cycles performed for each amplification

\begin{tabular}{|c|c|c|c|c|c|}
\hline \multirow[t]{2}{*}{ NOTCH1 (22 cycles) } & Fwd & CCCACTCATTCTGGTTGTCG & HES5 & Fwd & AGAAAAACCGACTGCGGAAG \\
\hline & Rev & CGCCTTTGTGCTTCTGTTCT & (26 cycles) & Rev & GACAGCCATCTCCAGGATGT \\
\hline \multirow[t]{2}{*}{ JAGGED1 ( 25 cycles $)$} & Fwd & GTCGGTCTTCCAGTCTCCAG & IFI-16 & Fwd & CCAGCTTTTCTGCTTTCGAC \\
\hline & Rev & TGATTTCCTTGATCGGGTTC & (24 cycles) & Rev & GTTGGTGGCATCTGAGGAGT \\
\hline \multirow[t]{2}{*}{ JAGGED2 ( 25 cycles $)$} & Fwd & AATGGTGGCATCTGTGTTGA & $M N D A$ & Fwd & CAAGCAAGCATCTGGAACAA \\
\hline & Rev & GCGATACCCGTTGATCTCAT & ( 25 cycles) & Rev & AGCTTGCGGTCAACTGTTCT \\
\hline \multirow[t]{2}{*}{ DLL-1 (26 cycles) } & Fwd & TATCCGCTATCCAGGCTGTC & MELTRIN $\beta$ & Fwd & TGCAGGAACACCTCCTTCTT \\
\hline & Rev & GGTGGGCAGGTACAGGAGTA & (26 cycles) & Rev & CATAGGCCCACTGTCGATAC \\
\hline \multirow[t]{2}{*}{$D L L-3$ (23 cycles) } & Fwd & TCCCAGAATTTCAAACCCAA & $I d 3$ & Fwd & ACTCAGCTT GCCAGGTGGA \\
\hline & $\operatorname{Rev}$ & TCACCTCCAATCTGGTCTCC & (23 cycles) & Rev & AAGCTCCTTTTGTCGTTGGA \\
\hline \multirow[t]{2}{*}{$D L L-4$ (25 cycles) } & Fwd & ACTACTGCACCCACCACTCC & HERP2 & Fwd & AACTGTTGGTGGCCTGAATC \\
\hline & Rev & CCTGTCCACTTTCTTCTCGC & (26 cycles) & Rev & GCTGGTAAATGCAGGCGTAT \\
\hline \multirow[t]{2}{*}{ HES1 (23 cycles) } & Fwd & ACGACACCGGATAAACCAAA & DELTEX & Fwd & CTTCCCTGATACCCAGACCA \\
\hline & Rev & CGGAGGTGCTTCACTGTCAT & (24 cycles) & Rev & CGTGCCGATAGTGAAGATGA \\
\hline \multirow[t]{2}{*}{$p T \alpha$ (23 cycles) } & Fwd & CTGGATGCCTTCACCTATGG & GAPDH & Fwd & CCATGGAGAAGGCTGGGG \\
\hline & Rev & CAGGTCCTGGCTGTAGAAGC & (20 cycles) & Rev & CAAAGTTGTCATGGATGACC \\
\hline
\end{tabular}

Amplified cDNAs were separated by agarose gel electrophoresis in the presence of ethidium bromide $(100 \mathrm{ng} / \mathrm{ml})$. Results of electrophoresis were acquired by the image acquisition system EDAS 2400 (Kodak) and following analysed by Kodak 1D image analysis system.

\subsection{Statistical analysis}

All values resulting from image analysis of PCR products were normalized against the corresponding values obtained from GAPDH amplification. The resulting values were then statistically analysed by ANOVA (analysis of variance) test to reveal if there is any statistical difference in gene expression associated with the different leukemia cell samples, T-ALL, BCP-ALL and AML. Analysis was independently performed for each gene. The tested hypothesis was:

$H_{0}=$ gene expression values are equal across the different cell types; versus

$H_{1}=$ at least two cell types gene expression values are different.

The rejection of the null hypothesis $\left(H_{0}\right)$ $(P$-value $<0.01)$ drives to the conclusion that the analysed gene expression levels are different in, at least, two among the analysed populations. A following multiple comparison test (Tukey test) was performed to determine which particular population differs from the others: the difference between values distributions of two samples groups is significant when $P<0.05$; is not significant when $P>0.05$.

\section{Results}

\subsection{Identification of NOTCH pathway target genes}

We have previously reported the concordance between HES1 and NOTCH1 genes expression both in T-cell ALL and B-cell lymphoma cell lines [12]. To identify further NOTCH pathway target
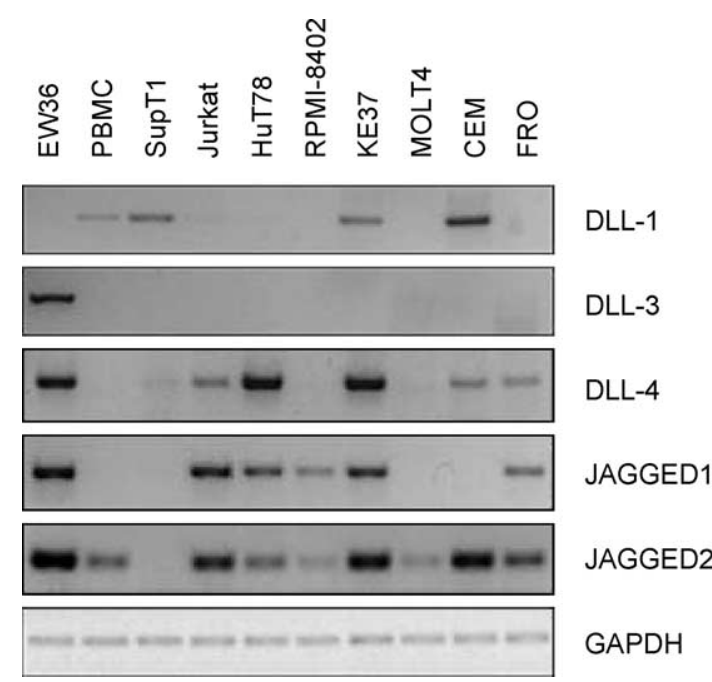

Fig. 1. RT-PCR analysis of DSL ligands expression in T-ALL cell lines; EW36 B cell line and PBMC have been used as control for $J A G G E D 1, J A G G E D 2, D L L-1, D L L-3, D L L-4$. To verify the specificity of the reactions, parallel amplifications have been performed on genomic DNA resulting in amplified DNA fragments of different size (not shown). The house keeping gene GAPDH has been used for normalization. 
genes we have expanded the analysis to the genes reported to be modulated by NOTCH pathway: $p T \alpha$ (the gene for preTCR- $\alpha$ ), DELTEX, HERP2, HES5, $M E L T R I N \beta$ (an ADAM family metallo-protease), IFI16 and MNDA [14,15]. Only the expression of $p T \alpha$ gene correlates with NOTCHI and HESI expression (data not shown). Accordingly, the subsequent analyses on primary leukemic cells were limited to NOTCH1, HES1 and $p T \alpha$ as marker of NOTCH1 signaling.

\subsection{NOTCH1 and ligands expression in T-ALL cell lines}

To assess a possible contribution of the ligands in the NOTCH pathway activation we performed RTPCR analyses on T-ALL leukemia cell lines of transcripts for all the DSL ligands. The following T-ALL cell lines were analysed: SupT1 carrying t(7;9)(q34;q34.3), Jurkat, HuT78, RPMI-8402, KE37, MOLT4, CEM, FRO. PBMC isolated from normal

T-ALL

$+-122344567891011121314151617181920212223242526272829303132$

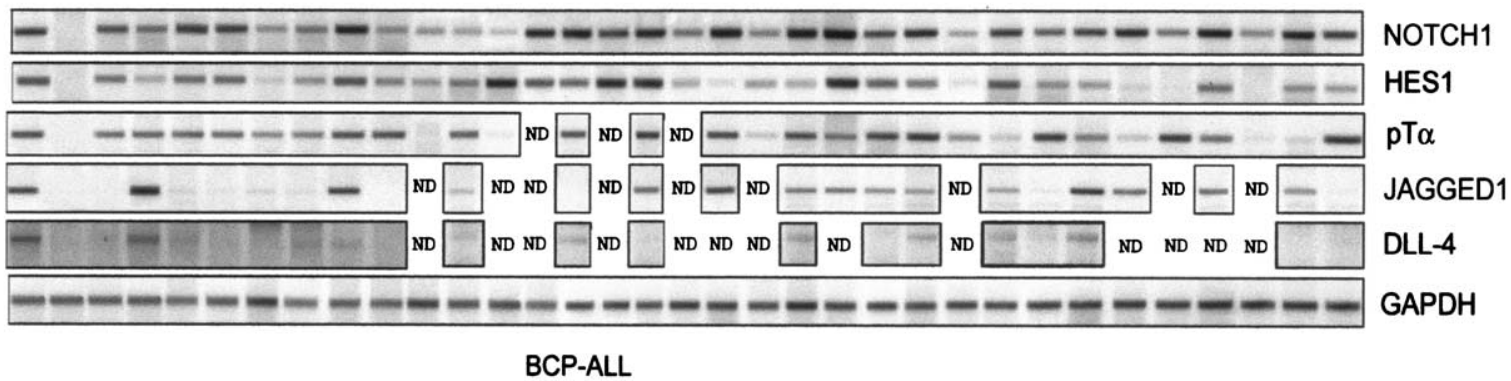

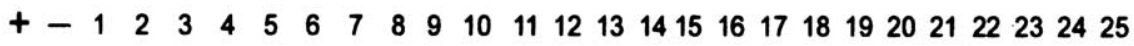

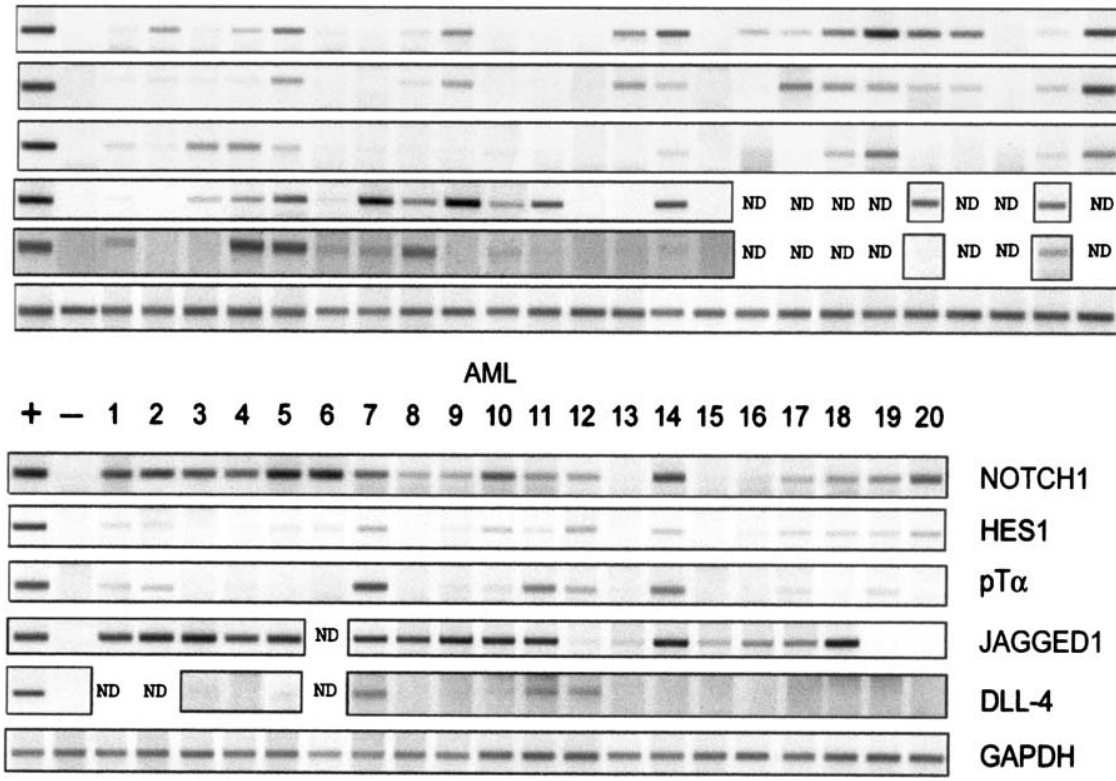

Fig. 2. RT-PCR analysis of Notch pathway-related genes, NOTCH1, its target genes, $H E S 1$ and $p T \alpha$, and the ligands $J A G G E D 1$ and $D L L-4$, in blood samples from patients with acute leukemia T-ALL, BCP-ALL and AML. To verify the specificity of the reactions, positive and negative controls were inserted: for the amplification of NOTCH1, HES1 and $p T \alpha$ positive and negative controls are, respectively, MOLT4 and BL41 cell lines, for JAGGED1 and DLL-4 amplifications EW36 and MOLT4 cell lines. The house keeping gene GAPDH has been used for normalization. Results of one representative experiment are reported. 
donors and the EW36 B lymphoma cell line were used as controls.

As shown in Fig. 1 all T-ALL cell lines, that we have previously assessed to be characterized by NOTCH1 pathway activation [12], expressed one or more NOTCH ligands encoding genes.

\subsection{Expression analysis of NOTCH pathway related genes on primary acute leukemia samples}

Fig. 2 shows the representative results of RT-PCR analysis of NOTCH1, HES1, pT $\alpha, J A G G E D 1$ and $D L L-4$ genes expression in primary ALL and AML samples and PBMC from normal donors. RT-PCR analysis was performed three independent times and showed reproducible results (data not shown).

Results of all experiments performed in $32 \mathrm{~T}-\mathrm{ALL}$, $25 \mathrm{BCP}-\mathrm{ALL}$ and $20 \mathrm{AML}$ patient samples are shown in Fig. 3. The box-plots used for presenting data show the median value (dark line) within a box containing $50 \%$ of the samples (25-75th percentile). Statistical analyses were performed by using ANOVA and Tukey tests. T-ALL samples showed NOTCH1 levels of expression equivalent to AML $(P>0.05)$, whereas they are higher if compared to BCP-ALL $(P<0.01)$. With respect to NOTCH1 pathway signaling, as measured by HES1 and $p T \alpha$ genes expression, T-ALL samples showed the highest levels of expression as compared to AML $(P<0.01)$ and furthermore in BCP-ALL samples $(P<0.01)$.

Finally, the analysis of the DSL ligands expression was performed for JAGGEDl and DLL-4. Primary samples from both B and T-cell leukemia showed comparable levels of mRNA expression. AML samples displayed the highest levels of JAGGEDI gene expression if compared to T-ALL $(P<0.01)$ and BCP-ALL $(P<0.05)$. No differences were detected in the three different leukemia subgroups with respect to $D L L-4$ gene expression (data not shown).

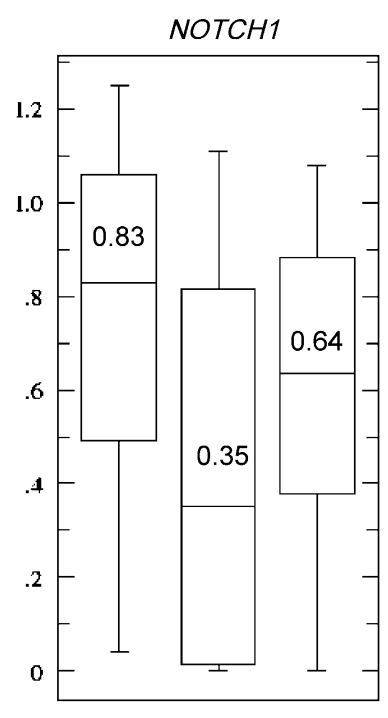

T-ALL BCP-ALL AML

T-ALL vs BCP-ALL $p<0.01$ T-ALL vs AML $p>0.05$ BCP-ALL vs AML $p>0.05$

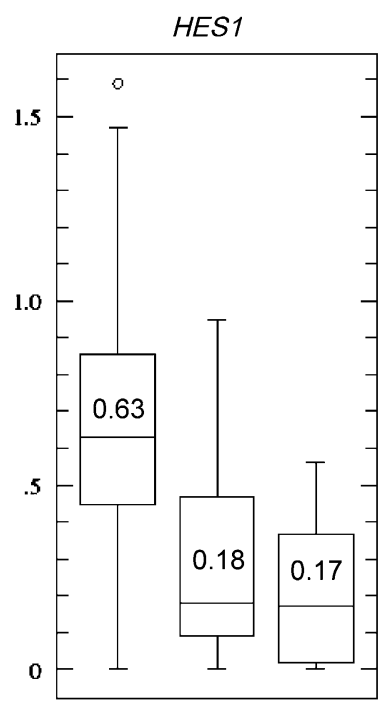

T-ALL BCP-ALL AML

T-ALL vs BCP-ALL $p<0.01$

T-ALL vs AML $p<0.01$ BCP-ALL vs AML $p>0.05$

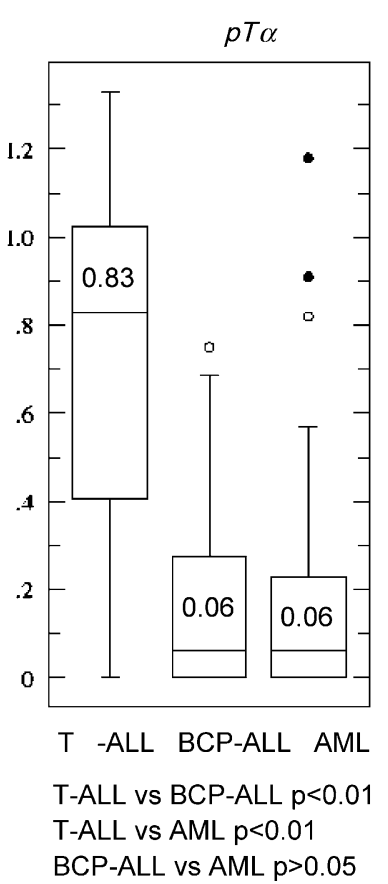

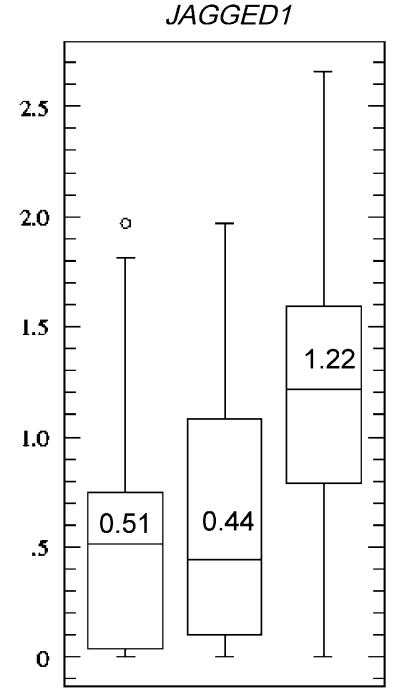

T -ALL BCP-ALL AML

T-ALL vs BCP-ALL $p>0.05$ T-ALL vs AML $p<0.01$ BCP-ALL vs AML $p<0.05$

Fig. 3. Median and distribution of the expression values of NOTCH related genes (NOTCH1, HES1, pT $\alpha$ and JAGGED1) measured in blood samples from patients with T-ALL, BCP-ALL and AML. Normalization has been performed against GAPDH gene expression. Box-plots have been used to represent data and the median value (dark line) is reported within a box containing 50\% of the samples (25-75th percentile). Results of Tukey test (multiple comparison) are reported: the difference between values distributions of two samples groups is significant when $P<0.05$; is not significant when $P>0.05$. 


\section{Discussion}

During T-cell lineage development NOTCHI expression is finely regulated [16]: NOTCH1 drives cell fate decision (the choice between TCR $\gamma / \delta$ or $\alpha / \beta$ and between $\mathrm{CD}^{+}{ }^{+}$or $\mathrm{CD}^{+}$) by inductive interactions from thymic stromal cells [17]; its expression is physiologically down-regulated to permit successful maturation by interfering with TCR signal strength [18]. NOTCH1 seems to play a role in differentiation also through its ability to affect cell proliferation by modulation of cell cycle [19] with the consequent accelerated progression through $\mathrm{G}_{1}$ phase: by maintaining cells in a proliferative state NOTCH1 signaling may interfere with differentiation. Furthermore, during T-cell lineage development, NOTCH1 plays a role in rescuing cells from apoptosis [20]. The present study confirms and further extends the role of the signaling driven by NOTCH1 and its ligands in the pathogenesis of different types of acute leukemia: T-ALL, BCP-ALL and AML. We show that high levels of NOTCH1 gene expression represent a common feature of T-ALL independently from the presence of the $t(7 ; 9)(\mathrm{q} 34 ; \mathrm{q} 34.3)$ translocation observed in less than $1 \%$ patients.

It has been suggested that the oncogenic ability of NOTCH1 truncated form may be due to the observed failure of NOTCH1 pathway down-regulation at the DP stage of $\mathrm{T}$ lineage development leading to a differentiation block at intermediate stages and malignant transformation proper of T-ALL [18]. The widespread over-signaling of NOTCH1 pathway relieved in T-ALL suggests that NOTCH pathway deregulation may represent a common way by which different genetic alterations lead to the deregulation of thymocytes development and to leukemogenesis. Recent evidences further support the hypothesis that several

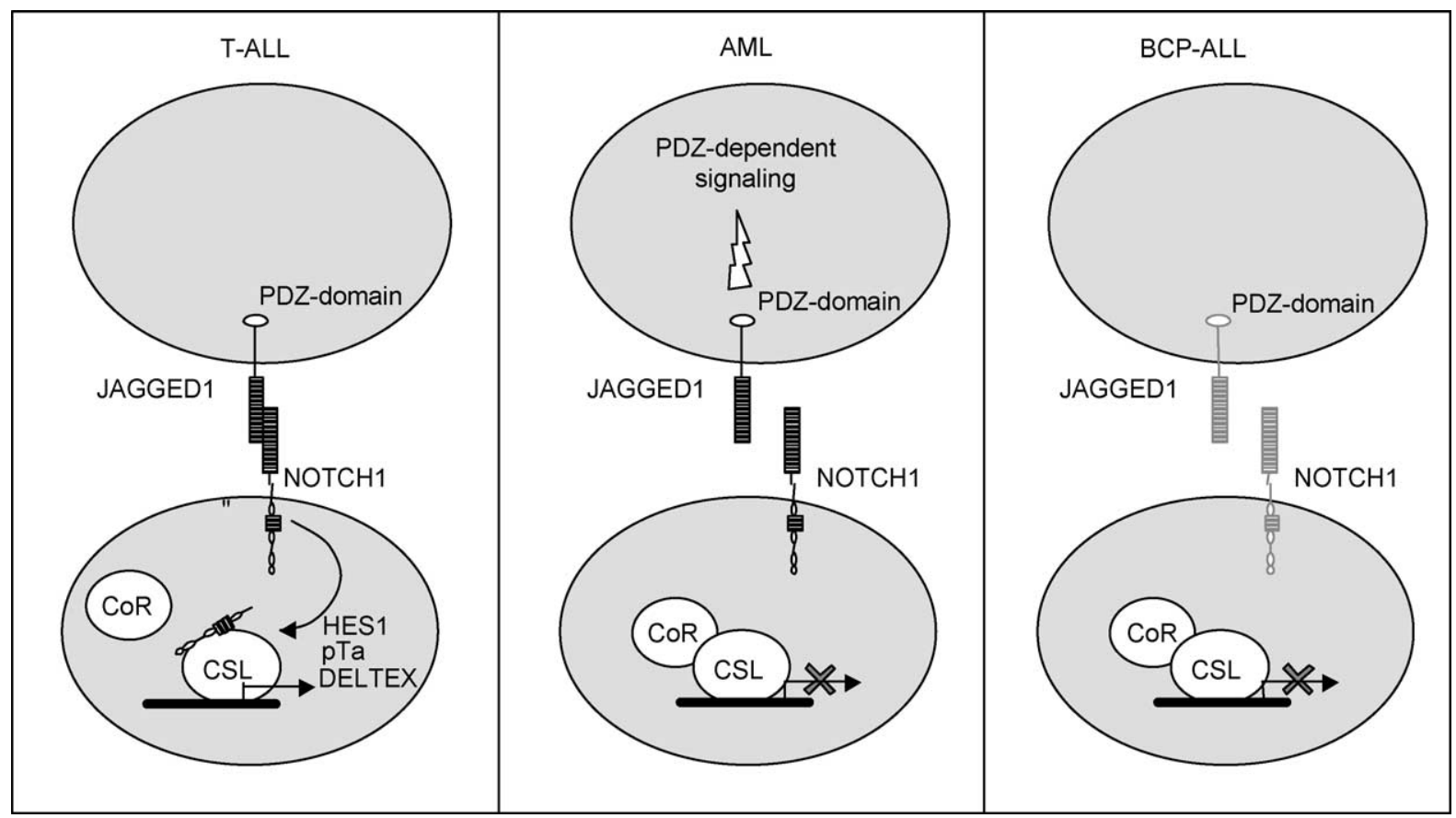

Fig. 4. A scheme of the possible bidirectional signaling of Notch1/DSL ligands. In T-ALL Jagged1, or other DSL ligands, activate NOTCH1 signaling and the transcription of target genes; on the contrary the high levels of JAGGED1 in AML do not produce the activation of NOTCH1 signaling, even if NOTCH1 is expressed on these cells, possibly because of a lack of sensibility of NOTCH1 to JAGGED1-induction due to a decreased binding affinity; on the other side in AML cells the signaling starting from the PDZ domain of JAGGED1, reported from other work, might contribute to leukemogenesis. In BCP-ALL low levels of both NOTCH1 and JAGGED1suggest they do not play any role in this neoplasia. The interaction between NOTCH1 and the DSL ligands, here represented on different cells, might also take place inside the same cell driving to an autocrine signaling. 
oncogenes, as IKAROS dominant negative form, $B M P 2$, the bHLH transcriptional regulators, TAL1, $T A L 2, L Y L 1, B H L H B 1$ and $L M O$ may play their well-assessed role in T-ALL genesis by acting upstream NOTCH1 pathway [21-24].

A possible role for NOTCH ligands in inducing high level of pathway activity was assessed through the analysis of JAGGEDI and DLL-4 transcription levels in acute leukemia blood samples. Both ligands were selected since $D L L-4$ overexpression in mouse bone marrow results in $\mathrm{T}$ Cell leukemia [9]. Moreover, JAGGED1 expression in stromal cells of bone marrow and thymus seems to drive haematopoietic differentiation by interacting with NOTCH1 in haematopoietic progenitors [6]. Finally, a possible role for JAGGED1 has been suggested for T-cell-derived anaplastic large cell lymphoma [10] and acute myelogenous leukemia [11].

We have found that JAGGEDI is highly expressed only in AML samples, thus confirming a selective role of this ligand in AML [11]. By contrast, DLL-4 generally showed low levels of expression without any evident differences among the different leukemia subgroups.

The high level of JAGGED1 expression suggests that this ligand might be important per se and not necessarily in relation to NOTCH pathway activation, according to the data recently reported by Ascano et al. [25] who demonstrated the existence of a NOTCH-independent pathway driven directly by JAGGED1: a scheme of NOTCH1/DSL ligands signaling in acute leukemia is reported in Fig. 4.

In conclusion the expression analysis of genes involved in NOTCH1 signaling suggests that NOTCH1 pathway exerts a wide role in acute leukemia. More specifically in T-ALL, its function is not restricted to those cases carrying the $\mathrm{t}(7 ; 9)(\mathrm{q} 34 ; \mathrm{q} 34.3)$ translocation, due to its central role in driving cell development. In AML our findings suggest a possible autonomous role of JAGGED1 in supporting AML growth.

\section{Acknowledgements}

This work was supported by grants from MIUR to $\mathrm{PC}$ and to $\mathrm{AB}$, from University of Milano (FIRST) to
$\mathrm{RC}$, to $\mathrm{PC}$ and to $\mathrm{AB}$, from Associazione Italiana per la Ricerca sul Cancro (AIRC) to AB.

\section{References}

[1] S. Kojika, J.D. Griffin, Notch receptors in hematopoiesis, Exp. Hematol. 29 (2001) 1041-1052.

[2] J.R. Shutter, S. Scully, W. Fan, W.G. Richards, J. Kitajewski, G.A. Deblandre, et al., D114, a novel Notch ligand expressed in arterial endothelium, Genes Dev. 14 (2000) 1313-1318.

[3] L.A. Milner, A. Bigas, Notch as a mediator of cell fate determination in haematopoiesis: evidence and speculation, Blood 93 (1999) 2431-2444.

[4] F. Radtke, A. Wilson, G. Stark, M. Bauer, J. van Meerwijk, H.R. MacDonald, M. Aguet, et al., Deficient T cell fate specification in mice with an induced inactivation of Notch1, Immunity 10 (1999) 547-558.

[5] H.T. Tan-Pertel, L. Walker, D. Browning, A. Miyamoto, G. Weinmaster, J.C. Gasson, Notch signaling enhances survival and alters differentiation of 32D myeloblasts, J. Immunol. 165 (2000) 4428-4436.

[6] L. Li, L.A. Milner, Y. Deng, The human homolog of rat Jagged 1 expressed by marrow stroma inhibits differentiation of $32 \mathrm{D}$ cells through interaction with Notch1, Immunity 8 (1998) 43-45.

[7] L.W. Ellisen, J. Bird, D.C. West, A.L. Soreng, T.C. Reynolds, S.D. Smith, et al., TAN-1, the human homolog of the Drosphila Notch gene, is broken by chromosomal translocations in T lymphoblastic neoplasms, Cell 66 (1991) 649-661.

[8] W.S. Pear, J.C. Aster, M.L. Scott, R.P. Hasserjian, B. Soffer, J. Sklar, et al., Exclusive development of T cell neoplasms in mice transplanted with bone marrow expressing activated Notch alleles, J. Exp. Med. 183 (1996) 2283-2291.

[9] X.-Q. Yan, U. Sarmiento, Y. Sun, G. Huang, J. Guo, T. Juan, et al., A novel Notch ligand, Dll4, induces T-cell leukemia/ lymphoma when overexpressed in mice by retroviral-mediated gene transfer, Blood 98 (2001) 3793-3799.

[10] F. Jundt, I. Anagnostopoulos, R. Forster, S. Mathas, H. Stein, B. Dorken, Activated Notch1 signaling promotes tumor cell proliferation and survival in Hodgkin and anaplastic large cell lymphoma, Blood 99 (2002) 3398-3403.

[11] S. Tohda, N. Nara, Expression of Notch1 and Jagged1 proteins in acute myeloid leukemia cells, Leuk. Lymphoma 42 (2001) $467-472$.

[12] R. Chiaramonte, E. Calzavara, F. Balordi, M. Sabbadini, D. Capello, G. Gaidano, et al., Differential regulation of Notch signal transduction in leukemia and lymphoma cells in culture, J. Cell. Biochem. 88 (2003) 569-577.

[13] P. Chomczynski, N. Sacchi, Single-step method of RNA isolation by acid guanidinium-thiocyanate-phenol-chloroform extraction, Anal. Biochem. 162 (1987) 156-159.

[14] J.W. Choi, C. Pampeno, S. Vukmanovic, D. Meruelo, Characterization of the transcriptional expression of Notch-1 signaling pathway members, Deltex and HES1, in developing mouse thymocytes, Dev. Comp. Immunol. 26 (2002) 575-588. 
[15] M.L. Deftos, E. Huang, E.W. Ojala, C.A. Forbush, M.J. Bevan, Notch1 signaling promotes the maturation of CD4 and CD8 SP thymocytes, Immunity 13 (2000) 73-84.

[16] R.P. Hasserjian, J.C. Aster, F. Davi, D.S. Weinberg, J. Sklar, Modulated expression of notch1 during thymocytes development, Blood 88 (1996) 970-976.

[17] T. Washburn, E. Schweighoffer, T. Gridley, D. Chang, B.J. Fowlkes, D. Cado, et al., Notch activity influences the alpha/beta versus gamma/delta $\mathrm{T}$ cell lineage decision, Cell 88 (1997) 833-843.

[18] D.J. Izon, J.A. Punt, L. Xu, F.G. Karnell, D. Allman, P.S. Myung, et al., Notch1 regulates maturation of $\mathrm{CD}^{+}$ and $\mathrm{CD} 8^{+}$thymocytes by modulating TCR signal strength, Immunity 14 (2001) 253-264.

[19] C. Ronchini, A.J. Capobianco, Induction of cyclin D1 transcription and CDK2 activity by Notch ${ }^{\text {ic }}$ : implication for cell cycle disruption in transformation by Notch ${ }^{\text {ic }}$, Mol. Cell. Biol. 21 (2001) 5925-5934.

[20] L. Shelly, C. Fuchs, L. Miele, Notch-1 inhibits apoptosis in murine erythroleukemia cells and is necessary for differentiation induced by hybrid polar compounds, J. Cell. Biochem. 73 (1999) 164.

[21] L.J. Beverly, A.J. Capobianco, Perturbation of Ikaros isoform selection by MLV integration is a cooperative event in Notch $^{\mathrm{IC}}$-induced T cell leukemogenesis, Cancer Cell 3 (2003) 551-564.

[22] L. Sun, M.L. Crotty, M. Sensel, H. Sather, C. Navara, J. Nachman, et al., Expression of dominant-Negative Ikaros Isoforms in T-cell acute lymphoblastic Leukemia, Clin. Cancer Res. 5 (1999) 2112-2120.

[23] T. Takizawa, W. Ochiai, K. Nakashima, T. Taga, Enhanced gene activation by Notch and BMP signaling cross-talk, Nucleic Acids Res. 31 (2003) 5723-5731.

[24] A.A. Ferrando, D.S. Neuberg, J. Stauton, L.L. Mignon, C. Huard, S.C. Raimondi, et al., Gene expression signatures define novel oncogenic pathways in T cell acute lymphoblastic leukemia, Cancer Cell 1 (2002) 75-97.

[25] J.M. Ascano, L.J. Beverly, A.J. Capobianco, The C-terminal PDZ-ligand of JAGGED1 is essential for cellular transformation, J. Biol. Chem. 278 (2003) 8771-8779. 